\title{
FMR1 Premutation and Demyelinating Syndromes: Case or Causality? A Case Report
}

\author{
Stefania Federica De Mercanti*, Marco Iudicello, Emanuele Franchin and Marinella Clerico \\ Clinical and Biological Sciences Department, Neurology Unit, Italy
}

*Corresponding author: Stefania Federica De Mercanti, Clinical and Biological Sciences Department, Neurology Unit, Torino, Italy

\begin{tabular}{|c|}
\hline ARTICLE INFO \\
\hline Received: February 09, 2019 \\
\hline Published: February 19, 2019 \\
\hline
\end{tabular}

Citation: Stefania Federica De M, Marco I, Emanuele F, Marinella C. FMR1 Premutation and Demyelinating Syndromes: Case or Causality? A Case Report. Biomed J Sci \& Tech Res 14(5)2019. BJSTR. MS.ID.002607.

Keywords: Multiple Sclerosis; Fragile $\mathrm{X}$-associated tremor/Ataxia Syndrome (FXTAS); X mental Retardation (FMR1); Central Nervous System (CNS); Middle Cerebellar PedunclesMCP

Abbreviations: MS: Multiple Sclerosis; FXTAS: Fragile X-Associated Tremor/Ataxia Syndrome; FMR1: X Mental Retardation; MRI: Magnetic Resonance Imaging; CSF: Cerebro Spinal Fluid; OB: Oligoclonal Bands

\section{ABSTRACT}

Purpose: This case report presents a patient with carrier status for Fragile X Syndrome and clinical and radiological features partially consistent with multiple sclerosis and with Fragile X-associated tremor/ataxia syndrome.

Methods: A 51-year-old woman, with a carrier status for Fragile X Syndrome with 68 CGG expansions, was referred to the neurological unit for suspected MS. At the age of 47 years patient rapidly developed motor symptoms with walking impairment and personality alterations. The first Magnetic Resonance Imaging showed white matter lesions consistent with demyelinating disorders; the cerebrospinal fluid analysis was negative for oligoclonal bands. Neurological disorders worsened a few years after diagnosis with onset of ataxia.

Discussion: Multiple sclerosis and Fragile X Syndrome have multiform neurological manifestations and the symptoms of the two diseases can be very similar to each other. The absence of oligoclonal bands does not exclude the diagnosis of multiple sclerosis a priori, the presence of white matter lesions can be found in both diseases. Regarding the molecular mechanism involved both conditions appear to be linked by the increase of a small heat shock protein, the $\alpha \mathrm{B}$-crystallin, which elevation observed in FXTAS may lead to enhanced predisposition to autoimmune diseases.

Conclusion: This case report shows the need for further clinical, radiological and molecular studies, able to establish the relations between the Fragile X premutation carrier status and demyelinating disorders.

\section{Introduction}

\section{Background}

Fragile X-associated tremor/ataxia syndrome (FXTAS) is a late onset neurodegenerative disorder that involves individuals with permutation alleles of the fragile X mental retardation (FMR1) gene [1]. Premutation alleles (from 55 to 200 CGG repeats) of the FMR1 gene are common, occurring approximately in 1/100-300 females and in $1 / 300-800$ males [2]. The full mutation is much rarer: $1 / 2500-4000[3,4]$. FXTAS penetrance varies within males and females: it is approximately $40 \%$ in males and 5\%-10\% in females $[5,6]$. The phenotype includes intention tremor, parkinsonism, ataxia, peripheral neuropathy, autonomic disfunction and cognitive impairment [7]. Demyelinating diseases of the central nervous system (CNS) are a heterogeneous group of diseases of the nervous system in which the myelin sheath of neurons is damaged [8]. Multiple sclerosis (MS) is the most common demyelinating disease of the CNS with an estimated prevalence worldwide of 2.3 million people [9]. In this report we describe a woman with FXTAS and a clinical and radiological presentation partially consistent with a demyelinating disease.

\section{Case Report}

A 51-year-old woman was referred to our neurological unit for suspected MS. In the family history patient reports 3 sisters with premature ovarian failure (POF), of which two FMR1 gene premutation carriers. She has one son with Fragile X Syndrome 
(FXS) with 200 CGG expansions. After the diagnosis in the child the patient was tested for carrier status, with evidence of 68 CGG expansions (activation ratio 0,428). At the age of 47 years she rapidly developed lower limbs motor disorders, particularly on the left side with walking impairment. Quite simultaneously she developed personality alterations, with consequent diagnosis of bipolar disorder. She started a therapy with antipsychotics and lithium salts. The first Magnetic Resonance Imaging (MRI) desplayed supratentorial and infratentorial white matter high intensities on $\mathrm{T} 2$ weighted images, not pathological gadolinium enhancement. The cerebrospinal fluid (CSF) analysis was negative for oligoclonal bands. Based on clinical symptoms, and in consideration of neuroradiological images, despite the lack of clinical and radiological diagnostic criteria, the patient received diagnosis of MS. She has never been treated with pulse steroids, immunomodulatory or immunosoppressive therapy. Motor and psychological deficit and MRI findings remained stable until May 2012, when paraparesis worsened and the patient became ataxic. Brain MRIs revealed mild diffuse atrophy and multiple foci of increased T2-weighted signal intensity supra-and infratentorial.

\section{Discussion}

This report describes a 51-year-old female with FXTAS and a radiological picture consistent with a demyelinating disease and a phenotype partially compatible with MS and FXTAS. The etiology of MS is still under discussion; most likely MS occurs as a result of a combination of genetic, environmental and infectious factors [10]. MS is a typical young adults disorder, more common in women [10]. MS takes several forms, with new symptoms occurring either in attacks or relapses (relapsing forms) or slowly accumulating over time (progressive forms) [11]. Between attacks, symptoms may disappear completely (remitting MS), but permanent neurological problems often persist, especially as the disease advances. Since MS can affect any white matter portion of the CNS, almost any neurological symptom and sign can appear with the disease, and often progresses to physical and cognitive disability [10]. The most commonly used diagnostic tools are MRI neuroimaging, CSF analysis and evoked potentials. Brain and spine MRI shows demyelination areas and the intravenously administration of Gadolinium can highlight active plaques [12]. Testing of CSF can provide evidence of chronic inflammation of the CNS, such as oligoclonal bands of IgG on electrophoresis; inflammation markers are found in $75-85 \%$ of people with MS $[12,13]$. FXTAS is a neurodegenerative disorder, identified in 2001 by Dr. Randi Hagerman; it affects individuals with permutation alleles of the FMR1 gene [1]. The classic FXTAS phenotype includes kinetic tremor and cerebellar gait ataxia in FMR1 premutation carriers over the age of 50 .

Penetrance is approximately $40 \%$ in males and 5\%-10\% in females [5,6]. MRI of FXTAS patients can show moderate to severe generalized brain atrophy with ventricular enlargement, specific cerebellar atrophy, and subcortical and/or pontocerebellar white matter lesions [14-16]. Approximately 60\% of males with FXTAS have white matter lesions or hyperintensities on T2-weighted MRI in the middle cerebellar peduncles (MCP), termed the "MCP sign", which is a major radiologic feature of FXTAS, but it has also been reported in other neurological conditions such as multiple system atrophy $[17,18]$, recessive ataxia [18] and acquired hepatocerebral degeneration [19]. Since the initial FXTAS description, the literature has been enriched by numerous case reports and studies that lead to more awareness about the disease characteristics describing phenotypes that vary from the original diagnostic criteria. Although FXTAS was originally described in male patients, females with FXTAS have now been reported. Recent case reports described female carriers with typical FXTAS [20], with FXTAS and spasmodic dysphonia [21] and with FXTAS and MS [2,22]. The hypothesised mechanism leading to FXTAS, involves a direct toxic gain-of-function of the FMR1 mRNA, likely through altered regulation of some protein that interact with the high levels of altered FMR1 mRNA [7].

In neurons and astrocytes throughout the brain and brainstem of FXTAS patients there are intranuclear inclusions, astrocyte activation, axonal retraction bulbs, axonal loss, and myelin loss $[23,24]$. The inclusions contain $\alpha \beta$-crystallin, MBP, lamin $\mathrm{A} / \mathrm{C}$ isoforms, and numerous other proteins [24]. According to this finding the toxic effects of FMR1 mRNA should result in the disruption of nuclear lamin $\mathrm{A} / \mathrm{C}$ architecture and formation of perinuclear $\alpha \beta$-crystallin aggregates in cultured neural cells [25]. $\alpha \mathrm{B}$-crystallin is a small heat shock protein, likely involved both in the normal dynamics of cytoskeletal proteins [26] and in maintenance of cell survival during cellular stress [27]. According to recent knowledge, it seems that the $\alpha \mathrm{B}$-crystallin is a protein also related to the pathogenesis of MS. It is, in fact, a key antigen in the development of MS [28]; the up regulation of $\alpha \mathrm{B}$-crystallin in patients with FXTAS could result in loss of immunologic tolerance, leading to predisposition or exacerbation of MS. Firthermore $\alpha \mathrm{B}$-crystallin is highly expressed during the early phases of MS relapses [28], and autoantibodies to $\alpha \mathrm{B}$-crystallin are found in the cerebrospinal fluid of patients with MS. It is postulated that they could potentially interfere with the protective role in reducing brain inflammation and brain cell death [29]. Finally, carrier women seems to be particularly susceptible to develop autoimmune disorders, including autoimmune thyroiditis and fibromyalgia [22].

\section{Conclusion}

Our patient had clinical and radiological features partially consistent with MS, but not enough to meet the revised Mc Donalds diagnostic criteria [12]. According to the diagnostic criteria for FXTAS [14] the patient appears to have a degree of probability to have FXTAS defined as "possible", given the presence of a Major clinical sign (gait ataxia) and of a minor radiological sign (white matter lesions in cerebral white matter). Several studies have postulated different clinical hypotheses that can explain the simultaneous presence of the FMR1 premutation genotype and of 
a demyelinating disease such as MS. First of all both conditions are very common and, therefore, it would not be rare to observe the two conditions in the same patients, expecially a female, simply by chance. Regarding the molecular biological point of view, the two conditions appear to be linked by the increase of a small heat shock protein, the $\alpha \mathrm{B}$-crystallin, which elevation observed in FXTAS may lead to enhanced predisposition to autoimmune diseases, by predisposing or exacerbating a picture of demyelination.

In the approach to the patients is also important to consider the clinical features of presentation and development of the disease. Our patient, in fact, in addition to a clinical picture characterized by motor deficit compatible with both situations, experienced also a rapid onset of psychiatric disorders requiring specific antipsychotic therapy. Therefore, in the diagnostic process, especially if the patient is a female with POF, in presence of alterations on MRI consistent with a demyelinating process and with also atypical symptoms we recommend to screen the patient for FXTAS. In conclusion, all the findings described in this report, shows the need for further clinical, radiological and molecular studies, able to establish the relations between the fragile $\mathrm{X}$ premutation carrier status and demyelinating disorders.

\section{References}

1. Hall DA, RJ Hagerman (2018) Fragile X-Associated Tremor/Ataxia Syndrome: Unmet Needs and a Path for the Future. Front Genet 9: 100.

2. Greco CM, F Tassone, D Garcia Arocena, Tartaglia N, Coffey SM, et al. (2008) Clinical and neuropathologic findings in a woman with the FMR1 premutation and multiple sclerosis. Arch Neurol 65(8): 1114-1116.

3. Dombrowski C, S Lévesque, ML Morel, Rouillard P, Morgan K, et al. (2002) Premutation and intermediate size FMR1 alleles in 10572 males from the general population: loss of an AGG interruption is a late event in the generation of fragile X syndrome alleles. Hum Mol Genet 11(4): 371-378.

4. Fernandez Carvajal I, P Walichiewicz, X Xiaosen, Pan R, Hagerman PJ, et al. (2009) Screening for expanded alleles of the FMR1 gene in blood spots from newborn males in a Spanish population. J Mol Diagnostics 11(4): 324-329.

5. Hagerman PJ and RJ Hagerman (2004) The Fragile-X Premutation: A Maturing Perspective. Am J Hum Genet 11(4): 371-378.

6. Coffey SM, K Cook, N Tartaglia, Flora Tassone, Danh V Nguyen, et al. (2008) Expanded clinical phenotype of women with the FMR1 premutation. Am J Med Genet Part A 146(8): 1009-1016.

7. Berry Kravis E, L Abrams, SM Coffey (2007) Fragile X-associated tremor/ ataxia syndrome: Clinical features, genetics, and testing guidelines. Movement Disorders 22(14): 2018-2030.

8. Love S (2006) Demyelinating diseases. J Clin Pathol 59(11): 1151-1159.

9. Browne P, D Chandraratna, C Angood, Tremlett H, Baker C, et al. (2014) Atlas of Multiple Sclerosis 2013: A growing global problem with widespread inequity. Neurology 83(11): 1022-1024.

10. Compston A, A Coles (2008) Multiple sclerosis. Lancet 372(9648): 15021517.

11. Lublin FD, SC Reingold, J a Cohen, Cutter GR, Sørensen PS, et al. (2014) Defining the clinical course of multiple sclerosis: The 2013 revisions
Defining the clinical course of multiple sclerosis The 2013 revisions. Neurology 83(3): 278-286

12. Yamout BI, R Alroughani (2018) Multiple Sclerosis. Semin Neurol 38(2): 212-225.

13. Link H, YM Huang (2006) Oligoclonal bands in multiple sclerosis cerebrospinal fluid: an update on methodology and clinical usefulness. J Neuroimmunol 180(1-2): 17-28.

14. Jacquemont S, RJ Hagerman, M Leehey, Jim Grigsby, Lin Zhang, et al. (2003) Fragile X Premutation Tremor/Ataxia Syndrome: Molecular, Clinical, and Neuroimaging Correlates. Am J Hum Genet 72(4): 869-878.

15. Brunberg JA, S Jacquemont, RJ Hagerman, Berry Kravis EM, Grigsby J, et al. (2002) Fragile X premutation carriers: characteristic MR imaging findings of adult male patients with progressive cerebellar and cognitive dysfunction. AJNR Am J Neuroradiol 23(10): 1757-1766.

16. Loesch DZ, K Kotschet, N Trost, Greco CM, Kinsella G, et al. (2011) White matter changes in basis pontis in small expansion FMR1 allele carriers with parkinsonism. Am J Med Genet Part B Neuropsychiatr Genet 156(4): 502-506

17. Schrag A, D Kingsley, C Phatouros, C Mathias, A Lees, et al. (1998) Clinical usefulness of magnetic resonance imaging in multiple system atrophy. J Neurol Neurosurg. Psychiatry 65(1): 65-71.

18. Storey E, MA Knight, SM Forrest, et al. (2005) Spinocerebellar ataxia type 20. Cerebellum.

19. Lee J, D Lacomis, S Comu, et al. (1998) Acquired hepatocerebral degeneration: MR and pathologic findings. Am J Neuroradiol.

20. Rodriguez Revenga L, J Pagonabarraga, B Gómez Anson, López Mourelo 0 , Madrigal I, et al. (2010) Motor and mental dysfunction in motherdaughter transmitted FXTAS. Neurology 75(15): 1370-1376.

21. Horvath J, PR Burkhard, M Morris, Armand Bottani (2007) Expanding the phenotype of fragile $\mathrm{X}$-associated tremor/ataxia syndrome: A new female case [9]. Movement Disorders 22(11): 1677-1678.

22.Zhang L, S Coffey, LL Lua, Greco CM, Schafer JA, et al. (2009) FMR1 premutation in females diagnosed with multiple sclerosis. Journal of Neurology, Neurosurgery and Psychiatry 80(7): 812-814.

23. Greco CM, RF Berman, RM Martin, Tassone F, Schwartz PH, et al. (2006) Neuropathology of fragile X-associated tremor/ataxia syndrome (FXTAS). Brain 129(1): 243-255.

24. Iwahashi CK, DH Yasui, HJ An, Greco CM, Tassone F, et al. (2006) Protein composition of the intranuclear inclusions of FXTAS. Brain 129(1): 256271.

25. Garcia Arocena D, PJ Hagerman (2010) Advances in understanding the molecular basis of FXTAS. Hum Mol Genet 19(1): 83-89.

26. Perng MD, L Cairns, IjP van den, Prescott A, Hutcheson AM, et al. (1999) Intermediate filament interactions can be altered by HSP27 and alphaB crystallin. J Cell Sci 112(13): 2099-2112.

27. Salvador Silva M, CS Ricard, OA Agapova, Yang P, Hernandez MR (2001) Expression of small heat shock proteins and intermediate filaments in the human optic nerve head astrocytes exposed to elevated hydrostatic pressure in vitro. J Neurosci Res 66(1): 59-73.

28. van Noort JM, AC van Sechel, JJ Bajramovic, el Ouagmiri M, Polman CH, et al. (1995) The small heat-shock protein alpha B-crystallin as candidate autoantigen in multiple sclerosis 375(6534): 798-801.

29. Ousman SS, BH Tomooka, JM van Noort, Wawrousek EF, O Connor KC, et al. (2007) Protective and therapeutic role for alphaB-crystallin in autoimmune demyelination 448(7152): 474-479. 


\section{ISSN: 2574-1241}

DOI: 10.26717.BJSTR.2019.14.002607

Stefania Federica De Mercanti. Biomed J Sci \& Tech Res

(C) (i) This work is licensed under Creative

Submission Link: https://biomedres.us/submit-manuscript.php

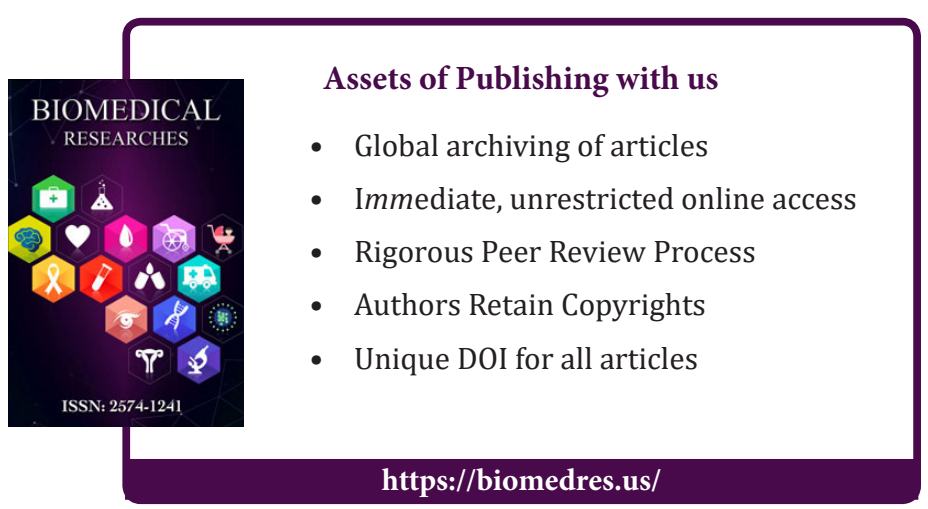

